\title{
ANCA-associated renal vasculitis and acute exacerbation of scleroderma-associated interstitial lung disease: A case report
}

\author{
Sandra Chartrand ${ }^{1,2}$, Elizabeth F.O. Kern ${ }^{1}$, David A. Taryle ${ }^{1}$, Aryeh Fischer ${ }^{1,3}$ \\ 1. Department of Medicine, National Jewish Health, Denver, Colorado, USA. 2. Department of Medicine, Hôpital \\ Maisonneuve-Rosemont affiliated to Université de Montréal, Montréal, Québec, Canada. 3. University of Colorado School \\ of Medicine, Aurora, Colorado, USA.
}

Correspondence: Aryeh Fischer. Address: 1775 Aurora Court, PO Box 6511, Mail Stop B115 Aurora, Colorado 80045, USA. Email: ARYEH.FISCHER@UCDENVER.EDU

Received: March 29, 2015

DOI : $10.5430 /$ crim.v2n2p89
Accepted: May 1, 2015

URL: http://dx.doi.org/10.5430/crim.v2n2p89

\begin{abstract}
In this report, we describe a patient with the unusual concomitant presentation of an acute exacerbation of systemic sclerosis-associated interstitial lung disease with the development of anti-neutrophil cytoplasmic antibody-associated active renal vasculitis. Systemic sclerosis is a connective tissue disease characterized by systemic autoimmunity and organ fibrosis and vasculopathy. Interstitial lung disease is identified in the majority of patients with systemic sclerosis and may have varying degrees of severity. Patients with systemic sclerosis-associated interstitial lung disease rarely develop acute exacerbations of interstitial lung disease. An overlap of systemic vasculitis has been reported to occur in patients with systemic sclerosis - yet this too is a rare occurrence. This case is unique in that it demonstrates the concomitant presentation of these rare clinical scenarios and serves to highlight the importance of a comprehensive and multidiscciplinary approach to the evaluation of patients in general, and systemic autoimmune patients in particular.
\end{abstract}

\section{Keywords}

Systemic sclerosis, Scleroderma, Interstitial lung disease, Acute exacerbation, ANCA-associated vasculitis

\section{I ntroduction}

Systemic sclerosis (SSc) is a potentially devastating connective tissue disease characterized by systemic autoimmunity and varying degrees of organ fibrosis and vasculopathy ${ }^{[1]}$. Interstitial lung disease (ILD) is the leading cause of death in SSc and is identified in the majority of patients with SSc. However, the ILD in SSc is not always progressive in nature and may not require specific therapy. Further, although patients with SSc-ILD are at risk of having acute exacerbations of ILD, this complication is relatively rare ${ }^{[1-9]}$. For unknown reasons, individuals with SSc may also be at risk for developing ANCA-associated vasculitis (AAV), though reliable estimates of the coincidence of these two systemic autoimmune conditions are lacking ${ }^{[10]}$. In this report, we describe an unusual concomitant presentation of an acute exacerbation of SSc-ILD along with development of isolated renal AAV in a patient with longstanding SSc. 


\section{Case presentation}

A 46-year-old woman with longstanding limited cutaneous SSc presented in October 2013 with pleurisy and exertional dyspnea. Over the previous 25 years, the patient's SSc had been characterized by an isolated positive antinuclear antibody (1:5120, nucleolar pattern), Raynaud's phenomenon, limited skin involvement, digital edema, telangiectasia, gastroesophageal reflux disease, and mild non-progressive ILD. The patient never required immunosuppressive therapies. Pulmonary function testing (PFT) results three months prior to presentation demonstrated mild and stable restrictive physiology (total lung capacity [TLC] 87\% predicted, forced vital capacity [FVC] 74\% predicted, forced expiratory volume in 1 second [FEV1] 78\% predicted, and diffuse lung capacity for carbon monoxide [ $\left.\mathrm{DL}_{\mathrm{CO}}\right]$ 73\% predicted (see Table 1).

Thoracic high-resolution computed tomography (HRCT) imaging two years prior revealed stable bibasilar fine reticulations and traction bronchiectasis without honeycombing, compatible with fibrotic nonspecific interstitial pneumonia (NSIP), with an estimated disease extent of 5\% (see Figure 1).

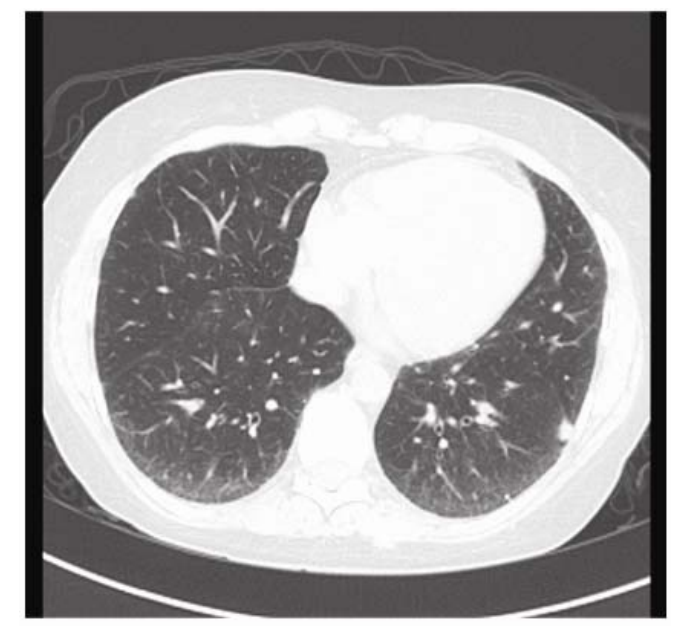

Figure 1. Thoracic imaging two years prior to presentation. HRCT scan image from October 2011 demonstrating bibasilar fine reticulations and traction bronchiectasis without honeycombing, of mild extent.

The patient's present illness consisted of three weeks of pleurisy, exertional dyspnea, and generalized fatigue. The review of systems was otherwise negative; specifically, there was no weight loss, fever, sweats, cough, sputum or musculoskeletal symptoms. On physical examination, the patient was in no acute distress. Ambient resting pulse oximetry was $93 \%$ but the patient's oximetry declined to $87 \%$ with ambulation. The patient was normotensive, without tachypnea or tachycardia. Cardiac examination was normal, without signs to suggest right heart compromise and there was no pericardial rub. Lung auscultation revealed diminished breath sounds and inspiratory crackles at the lower lung zones bilaterally. Laboratory tests were notable for markedly elevated inflammatory markers (erythrocyte sedimentation rate [ESR] at $122 \mathrm{~mm} / \mathrm{H}$ [normal $<30 \mathrm{~mm} / \mathrm{H}$ ] and C-reactive protein [CRP] at $20.32 \mathrm{mg} / \mathrm{dL}$ [normal $<0.4 \mathrm{mg} / \mathrm{dL}$ ]) and a normal white blood cell count. The echocardiogram was normal. Thoracic CT angiography was negative for pulmonary embolism but showed new bilateral lower lobe predominant consolidations along with extensive patchy and nodular ground glass opacities (GGO) (see Figure 2).

Over the next two days the patient developed fevers of $>38.0{ }^{\circ} \mathrm{C}$ and worsening dyspnea and required hospitalization. The patient underwent bronchoscopy and the bronchoalveolar lavage (BAL) cell count showed $43 \%$ macrophages, $40 \%$ neutrophils, $16 \%$ lymphocytes and $1 \%$ eosinophils, without evidence of alveolar hemorrhage. The lavage was not purulent and the gram stain did not show any organisms. Extensive serologic and BAL microbiology assessments for bacterial, viral, mycobacterial, fungal, and Pneumocystis jirovecii infection were negative. Empiric treatment with intravenous 
broad-spectrum antibiotics and pulse-dose methylprednisolone (1000 mg/day, for three consecutive days) was initiated. Laboratory assessment showed normal renal function, normal urinalysis, and high-titer C-ANCA and anti-MPO antibodies (see Table 1). Due to diagnostic uncertainty, concerns for pulmonary vasculitis, and the extent and severity of the parenchymal lung abnormalities, a surgical lung biopsy was performed. Lung histopathology demonstrated large regions of diffuse septal expansion by lymphocytes, plasma cells, edema and collagen and few airspace collections of fibrin and organizing fibrin compatible with a recent ILD exacerbation on a background of fibrotic NSIP. There was no evidence of pulmonary vasculitis. The patient improved clinically, antibiotics were discontinued and the patient was discharged on a daily oral dose of $40 \mathrm{mg}$ of prednisone.

The patient continued to improve clinically and thoracic HRCT scan one month after discharge confirmed complete resolution of GGO and parenchymal consolidations (see Figure 3).

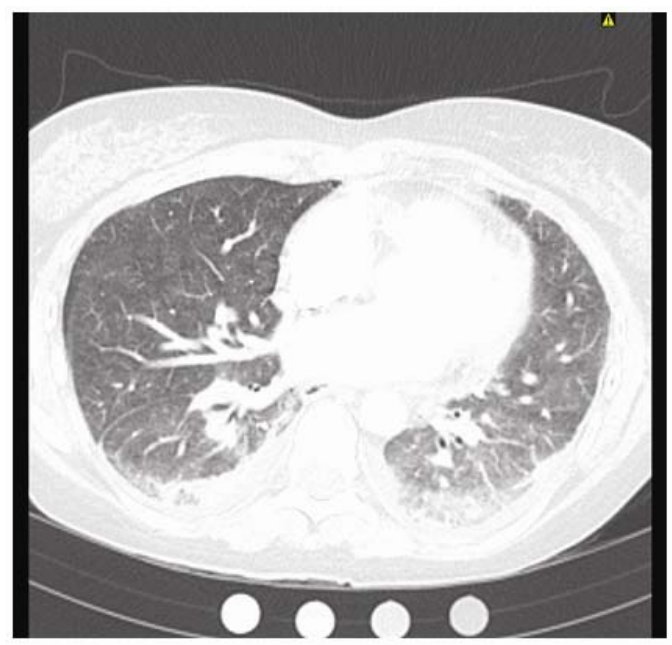

Figure 2. Thoracic imaging at presentation of acute exacerbation. Thoracic CT angiograpy scan image from October 2013 demonstrating bilateral lower lobe predominant consolidations along with extensive patchy and nodular ground glass opacities.

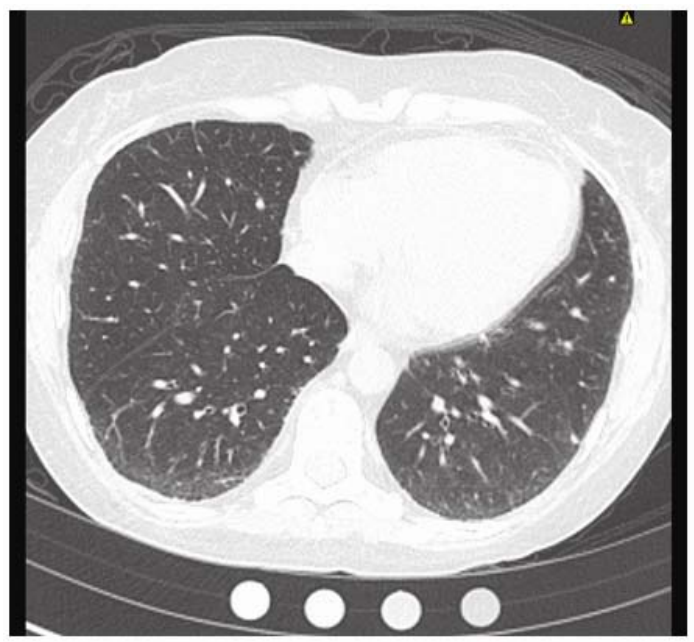

Figure 3. Thoracic imaging after treatment. Thoracic HRCT scan image from December 2013 demonstrating complete resolution of consolidations and ground glass opacities. Mild bibasilar fine reticulations and traction bronchiectasis without honeycombing, compatible with fibrotic nonspecific interstitial pneumonia, are still visible.

Repeat ANCA testing was positive for P-ANCA but negative for MPO and PR3 autoantibodies. Clinical review was negative for AAV symptoms or signs other than an abnormal urinalysis with proteinuria and significant microscopic hematuria (see Table 1). Due to concerns for an active renal vasculitis - and because the identification of renal vasculitis would impact the patient's treatment - a percutaneous renal biopsy was performed. The renal histopathology confirmed pauci-immune crescentic glomerulonephritis consistent with active renal vasculitis (see Figure 4).

In light of the finding of active renal AAV, the patient was treated with rituximab $375 \mathrm{mg} / \mathrm{m}^{2}$ weekly for four consecutive weeks with a tapering regimen of prednisone.

By April 2014, the patient was back to baseline respiratory status, prednisone was at $10 \mathrm{mg} / \mathrm{day}$, PFT values were back to baseline, renal function had stabilized and urinalysis normalized (see Table 1). Azathioprine at a target dose of $2 \mathrm{mg} / \mathrm{kg} /$ day was added for maintenance therapy of isolated renal AAV. 
Table 1. Laboratory and pulmonary function test results over time

\begin{tabular}{|c|c|c|c|}
\hline & October 2013 & December 2013 & April 2014 \\
\hline Erythrocyte sedimentation rate $(\mathrm{mm} / \mathrm{H})$ & 122 & 24 & 18 \\
\hline C-reactive protein (mg/dL) & 20.32 & 0.11 & 0.09 \\
\hline Creatinine (mg/dL) & 0.7 & 0.8 & 0.93 \\
\hline Glomerular filtration rate (mL/min) & 90 & 77 & $65^{*}$ \\
\hline Urine analysis & Normal & $\begin{array}{l}\text { Proteinuria 3+ } \\
\text { >50 RBC/HPF }\end{array}$ & Normal \\
\hline ANCA by immunofluorescence & C-ANCA 1:320 & P-ANCA 1:1280 & Not done \\
\hline ANCA by ELISA & $\begin{array}{l}\text { MPO >100 U; } \\
\text { PR3 negative }\end{array}$ & $\begin{array}{l}\text { MPO negative; } \\
\text { PR3 negative }\end{array}$ & $\begin{array}{l}\text { MPO negative; } \\
\text { PR3 } 57 \text { U } \\
\text { (moderate titer positive) }\end{array}$ \\
\hline Pulmonary function testing & (August 2013) & & \\
\hline FVC, L (\% predicted) & $2.59(74)$ & $1.89(55)$ & $2.44(72)$ \\
\hline FEV1, L (\% predicted) & $2.26(78)$ & $1.75(64)$ & $2.08(76)$ \\
\hline $\mathrm{DL}_{\mathrm{CO}}, \mathrm{mL} / \mathrm{min} / \mathrm{mmHg}$ (\% predicted) & $18.98(73)$ & $20.03(92)$ & $20.04(92)$ \\
\hline TLC, L (\% predicted) & $4.11(87)$ & $3.60(75)$ & $4.07(85)$ \\
\hline
\end{tabular}

Note. ANCA: Anti-neutrophil cytoplasmic antibody; DLCO: Diffuse lung capacity for carbon monoxide; FVC: Forced vital capacity; FEV1: Forced expiratory volume in 1 second; MPO: Myeloperoxidase; PR3: Proteinase 3; RBC/HPF: Red blood cell by high power field; TLC: Total lung capacity. * Decreased to 48 mL/min in March 2014.

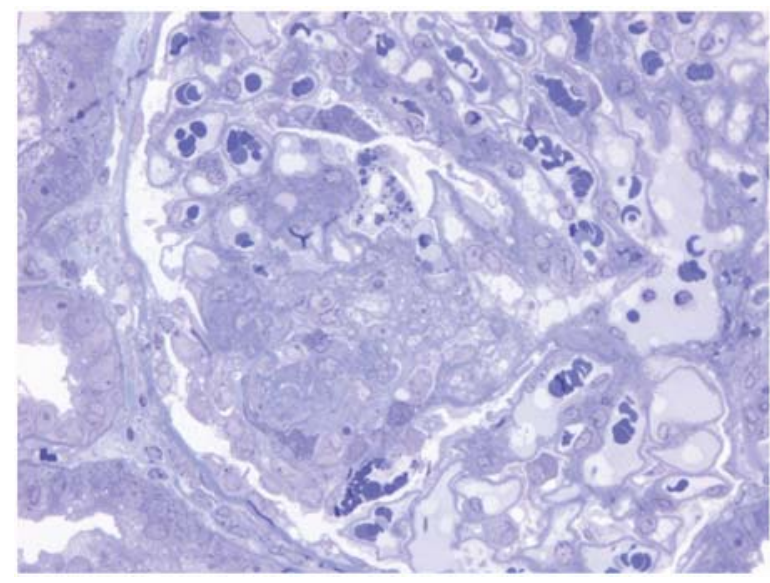

Figure 4. Electron microscopy image of glomerular tissue with evidence of crescentic glomerulonephritis, consistent with active renal vasculitis. Image courtesy of Nephropath (Little Rock, AR, USA).

\section{Discussion}

In this report we describe a case of isolated renal AAV that developed during an acute exacerbation of SSc-ILD. Acute exacerbations of ILD have been described with SSc ${ }^{[2]}$ but this complication occurs less frequently and tends to respond more favorably than with idiopathic pulmonary fibrosis (IPF) $\left(1.25 \%-3.3 \% /\right.$ year) ${ }^{[2-4]}$. The proposed criteria for diagnosis of acute exacerbation includes: a prior or concurrent diagnosis of a chronic ILD, worsening in dyspnea lasting less than a month, hypoxemia, new ground glass opacities on thoracic HRCT scan and exclusion of other causes ${ }^{[5]}$. Histopathology of acute exacerbation typically reveals the injury pattern of diffuse alveolar damage ${ }^{[3]}$. Risk factors include advanced age ${ }^{[2]}$, 
recent lung biopsy ${ }^{[3]}$, severe ILD (but also described in mild cases) and usual interstitial pneumonia pattern ${ }^{[3]}$. Quiescent extra-thoracic features do not preclude the occurrence of acute exacerbation in CTD-ILD. Similar to acute exacerbations in IPF, high dose corticosteroid therapy is the mainstay of therapy for acute exacerbation in connective tissue disease associated-ILD ${ }^{[6]}$ and corticosteroid-sparing therapies are commonly employed but their efficacy within this context is unknown $^{[7-9]}$.

The association between SSc and AAV was first reported in $1994^{[11]}$ and since then, more than 50 cases have been described in the literature ${ }^{[10,12,13]}$. It is not known why certain individuals with SSc develop an overlap of AAV and no reliable risk factors for this occurrence have been identified. The combination of these disorders is uncommon with prevalence estimates ranging from $1.3 \%$ for clinically expressed $\mathrm{AAV}^{[14]}$ to $2.8 \%$ for ANCA positivity alone ${ }^{[10]}$. From the reported cases, the AAV presents 8 to 9.5 years after the SSc onset, and is more commonly seen in women in their 5th or 6th decade. ILD was recognized in most cases (70\%), as was gastrointestinal tract involvement (68\%). There is no predilection to limited vs. diffuse cutaneous involvement ${ }^{[10,12,13]}$. Anti-topoisomerase I (Scl-70) antibody is positive in $70 \%$ and anti-centromere antibody in $14 \%-20 \%$ of cases. The most common AAV manifestations in those with SSc are diffuse alveolar hemorrhage and pauci-immune glomerulonephritis. These manifestations occur in combination in $30 \%-60 \%$ of cases, and isolated renal involvement is noted in $40 \%-70 \%{ }^{[10,12,13]}$. The majority of cases are P-ANCA and MPO antibody positive (72\%) but as many as $24 \%$ are P-ANCA and PR3 antibody positive ${ }^{[10,12,13]}$.

Acute exacerbations of ILD can occur even in mild SSc-ILD, should prompt thorough evaluations to exclude infectious or other etiologies for the respiratory compromise, and may be responsive to high-dose corticosteroids. AAV may rarely coincide with SSc and typically manifests with diffuse alveolar hemorrhage or isolated renal vasculitis. The case described is particularly unique in the temporal relationship between the onset of her acute ILD exacerbation and her developing AAV. This case also highlights the importance of a comprehensive and multidisciplinary approach to the evaluation of patients in general, and systemic autoimmune patients in particular.

\section{References}

[1] Wells AU, Steen V, Valentini G. Pulmonary complications: one of the most challenging complications of systemic sclerosis. Rheumatology (Oxford). 2009 Jun; 48 Suppl 3: iii40-4. PMid: 19487223 http://dx.doi.org 10.1093/rheumatology/kep109

[2] Suda T, Kaida Y, Nakamura Y, et al. Acute exacerbation of interstitial pneumonia associated with collagen vascular diseases. Respiratory Medicine. 2009; 103(6): 846-53. PMid: 19181509 http://dx.doi.org/10.1016/j.rmed.2008.12.019

[3] Park IN, Kim DS, Shim TS, et al. Acute exacerbation of interstitial pneumonia other than idiopathic pulmonary fibrosis. Chest. 2007; 132(1): 214-20. PMid: 17400667

[4] Solomon JJ, Fischer A. Connective Tissue Disease-Associated Interstitial Lung Disease: A Focused Review. J Intensive Care Med. 2013 Dec 25. PMid: 24371251

[5] Collard HR, Moore BB, Flaherty KR, et al. Acute exacerbations of idiopathic pulmonary fibrosis. Am J Respir Crit Care Med. 2007; 176(7): 636-43. PMid: 17585107

[6] Raghu G, Collard HR, Egan JJ, et al. An official ATS/ERS/JRS/ALAT statement: idiopathic pulmonary fibrosis: evidence-based guidelines for diagnosis and management. Am J Respir Crit Care Med. 2011; 183(6): 788-824. PMid: 21471066 http://dx.doi.org/10.1164/rccm.2009-040GL

[7] Parambil JG, Myers JL, Ryu JH. Diffuse alveolar damage: uncommon manifestation of pulmonary involvement in patients with connective tissue diseases. Chest. 2006; 130(2): 553-8. PMid: 16899858

[8] Ogawa D, Hashimoto H, Wada J, et al. Successful use of cyclosporin A for the treatment of acute interstitial pneumonitis associated with rheumatoid arthritis. Rheumatology (Oxford). 2000; 39(12): 1422-4. PMid: 11136888

[9] Tanaka F, Origuchi T, Migita K, et al. Successful combined therapy of cyclophosphamide and cyclosporine for acute exacerbated interstitial pneumonia associated with dermatomyositis. Intern Med. 2000; 39(5): 428-30. PMid: 10830189

[10] Quemeneur T, Mouthon L, Cacoub P, et al. Systemic vasculitis during the course of systemic sclerosis: report of 12 cases and review of the literature. Medicine (Baltimore). 2013; 92(1): 1-9. PMid: 23263715

http://dx.doi.org/10.1097/MD.0b013e31827781fd 
[11] Endo H, Hosono T, Kondo H. Antineutrophil cytoplasmic autoantibodies in 6 patients with renal failure and systemic sclerosis. J Rheumatol. 1994; 21(5): 864-70. PMid: 8064727

[12] Arad U, Balbir-Gurman A, Doenyas-Barak K, et al. Anti-neutrophil antibody associated vasculitis in systemic sclerosis. Semin Arthritis Rheum. 2011; 41(2): 223-9. PMid: 21277620 http://dx.doi.org/10.1016/j.semarthrit.2010.11.001

[13] Rho YH, Choi SJ, Lee YH, et al. Scleroderma associated with ANCA-associated vasculitis. Rheumatol Int. 2006; 26(5): 465-8. PMid: 16012875

[14] Derrett-Smith EC, Nihtyanova SI, Harvey J, et al. Revisiting ANCA-associated vasculitis in systemic sclerosis: clinical, serological and immunogenetic factors. Rheumatology (Oxford). 2013;52(10):1824-31. PMid: 23832959

http://dx.doi.org/10.1093/rheumatology/ket213 